\title{
VARIABLES COGNITIVAS Y METACOGNITIVAS EN LA COMPRENSIÓN DE TEXTOS CIENTIFICOS: EL PAPEL DE LOS ESQUEMAS Y EL CONTROL DE LA PROPIA COMPRENSION
}

\author{
OTERO, J. \\ Departamento de Física. Universidad de Alcalá. Alcalá de Henares.
}

\section{SUMMARY}

This paper tries to apply some models and results of psychological research on comprehension processes to the learning of scientific texts. More specifically, the role of the learner's schemes and control over self-comprehension when processing a scientific text is studied.

En algunos sistemas educativos, como el norteamericano, se ha constatado que "... los libros de texto ejercen un dominio abrumador en la experiencia de aprendizaje de la ciencia" (Harms 81, p. 115). En un 92\% de las clases de ciencias de los niveles equivalentes al bachillerato se utilizaban a finales de los años 70 uno $(48 \%)$ o más libros $(44 \%)$ de texto; los porcentajes de uso eran también del $90 \%$, o superiores, en Ia enseñan$z$ a básica, a partir del $4^{\circ}$ curso (Weiss 78, p. 89). En el sistema de enseñanza de las ciencias en España los porcentajes de uso son también probablemente altos. De acuerdo con datos referidos solamente a centros públi$\cos$ y privados de bachillerato de Madrid capital, un $91 \%$ de los profesores de Física y Química de $2^{\circ}$ de BUP y un $87 \%$ de los de $3^{\circ}$, declaran en el curso 1980 81 que se utilizaban libros de texto en sus clases (Velarde y Otero 84).

Desde hace años se investiga en las características de los libros de texto de ciencias. Estos estudios se fundamentan, en ocasiones, en alguna teoría sobre la forma en que los alumnos aprenden. Prosser 79), y Aguirre de Cárcer 83), por ejemplo, estudian la "demanda cognitiva" de los textos científicos basando su análisis en la teoría de Piaget. Otros investigadores, como Doran y Sheard 74), Fernández Uría 78), o Bullejos de la Higuera 83), simplemente examinan características de los textos, como la legibilidad, los contenidos, o las actividades, que se suponen con influencia en la calidad del texto como instrumento de enseñanza y aprendizaje, aunque esta suposición no esté fundamentada en teoría alguna, Entre ambos extremos se sitúan una variedad de estudios con mayor o menor fundamentación teórica. Por ejemplo, Finley 83 ) estudia el recuerdo de la información adquirida a partir de un texto, Williams y Yore 85), la legibilidad de los textos, (Mayer 85a, 85b), técnicas de análisis de su estructura y formas de mejorar su inteligibilidad, y Wandersee (88), la forma en que los alumnos extraen signíficado de elios.

Una de las formas en que se utiliza el libro de texto es como fuente de información a través de la lectura directa. Los estudios sobre este uso del libro de texto pueden aprovechar los resultados de la investigación en un área de gran actividad dentro de la psicolinguística: la comprensión de textos. En este artículo se intentan aplicar algunos modelos y resultados de las investigaciones psicológicas sobre los procesos de comprensión al aprendizaje de los textos científicos. Se estudian, en concreto, dos problemas que se han tratado en las investigaciones de esta clase: el papel de los esquemas del que aprende, y el control de la propia comprensión al procesar un texto científico. Los problemas de la primera clase son de naturaleza cognitiva mientras que los últimos pertenecen al área de la "metacognición". Los problemas metacognitivos, tratados extensamente, por ejemplo, por los investigadores sobre la lectura, empiezan a tomarse en consideración también en la enseñanza de las ciencias (Baird 86, Baker, en prensa). Flavell (76) ofrece una definición clásica del concepto de metacognición: 
"La metacognición se refiere al conocimiento que uno tiene sobre los propios procesos y productos cognitivos o sobre cualquier cosa relacionada con ellos, es decir, las propiedades de la información o los datos relevantes para el aprendizaje. Por ejemplo, estoy implicado en metacognición (metamemoria, metaaprendizaje, metaatención, metalenguaje, etc.) si me doy cuenta de que tengo más problemas al aprender A que al aprender B, si se me ocurre que debo comprobar $C$ antes de aceptarlo como un hecho... La metacognición se refiere, entre otras cosas, ai control activo y la orquestación y regulación subsiguiente de estos procesos..." (p. 232).

Brown (80) explica que: "...las destrezas de la metacognición son las que se atribuyen al ejecutivo en muchas teorías de la memoria humana e inteligencia artificial: predecir, comprobar, controlar, contrastar con la realidad y coordinar y controlar los intentos deliberados para estudiar, aprender o resolver problemas" (pag. 454).

Brown $(87$, p. 67) apunta también que el término metacognición ha sido atilizado en dos sentidos: para referirse al conocimiento que se puede tener sobre la propia actividad de conocer (saber, por ejemplo, qué hace que algo sea fácil o difícil de memorizar) y para referirse a las actividades de control y regulación de la cognición (por ejemplo, a las estrategias utilizadas para restablecer la comprensión, ante las dificultades encontradas en un texto difícil). En la última parte de este trabajo se consideran problemas metacognitivos en esta segunda acepción del término.

\section{EL PAPEL DE LOS ESQUEMAS}

Un hallazgo bien fundamentado por las investigaciones sobre el aprendizaje de textos, desde el trabajo pionero de Bartlett (32), es la influencia de los conocimientos que ya posee el sujeto en la adquisición de nueva información. Ausubel (68, Ausubel y otros 78) hace de este resultado un elemento central de su teoría del aprendizaje significativo (1). La investigación sobre el aprendizaje de las ciencias ha reflejado también la preocupación por el papel del conocimiento previo: el aprendizaje de los contenidos cientificos se ha visto que depende de las preconcepciones del alumno sobre los temas objeto de estudio. Una parte importante del esfuerzo de los investigadores ha estado dirigido a identificar estas preconcepciones en diversas áreas de la ciencia.

Diversos investigadores, dentro de la psicología cognitiva, han estudiado la función del conocimiento previo en la adquisición de nueva información postulando estructuras de conocimiento, con características similares, en las que se condensa el conocimiento general: guiones o "scripts" (Schank y Abelson 77), esquemas (Rumelhart y Ortony 77; Rumelhart 80), o marcos (Minsky 75). Todas estas nociones comparten la suposición de que el conocimiento está organizado en paquetes que se aplican en el proceso de comprensión. Un esquema, por ejemplo, se define como:

"...una estructura de datos que sirve para representar Ios conceptos genéricos almacenados en la memoria... Hay, creemos, al menos cuatro características esenciales de los esquemas que se combinan para convertirlos en un medio poderoso de representación del conocimiento en la memoria. Estas características son: 1) los esquemas tienen variables; 2) los esquemas pueden encajarse unos dentro de otros; 3) los esquemas representan conceptos genéricos que, tomados en conjunto, varían en nivel de abstracción; 4) los esquemas representan conocimiento, más que definiciones." (Rumelhart y Ortony, op. cit., p. 101).

Los esquemas juegan un papel fundamental en la comprensión. Un sujeto entiende una situación cuando es capaz de activar un esquema en el que se pueda integrar la información que se le proporciona. La proposición "Luis Miguel y Rosina comieron percebes" se entiende fácilemte al activar un esquema correspondiente a comer. Este esquema incluiría, al menos, variables o "casilias" correspondientes al sujeto, con valores normalmente limitados a personas o animales, y al objeto, con valores normalmente limitados a ciertas clases de materias comestibles. En este caso se comprueba que Luis Miguel y Rosina son previsiblemente nombres de personas, resultando un valor aceptable de la variable "sujeto" y que percebe corresponde a un objeto comestible, resultando un valor aceptable de la variable "objeto". Si la frase siguiente fuese "Dejaron una buena propina y se levantaron", quizá la mención de "propina" activase el esquema (o guión) más específico comer en restaurante. Una de las variables de este esquema corresponde a la acción de pagar. Aunque no se proporciona información sobre esta acción, el esquema permite darle un valor por defecto, suponiendo que han pagado, en metálico, o con una tarjeta de crédíto, antes de dar la propina. Téngase en cuenta que ésta es realmente una inferencia que se realiza sobre la base del esquema. Podría ser que, por alguna razón, les hubiese invitado el dueño del restaurante y únicamente dejasen propina al camarero que les atendió. El esquema permite hacer inferencias para que el discurso resulte coherente, es decir, las distintas proposiciones se vean relacionadas.

Un enunciado como "El desayuno fue delicioso porque los hilos estaban pegajosos" 2), tiene mayores dificultades de comprensión. La activación del esquema comer, debido a la mención de desayuno, no permite colocar fácilmente la información "los hilos estaban pegajosos" en ninguna de las casillas del esquema. Aunque tanto el léxico como la sintaxis son perfectamente accesibles, es difícil comprender la proposición si no se activa algún esquema relacionado con araña.

Aunque la concepción de esquema se encuentra sometida a críticas por sus limitaciones como explicación del proceso de comprensión (véase por ejemplo, Van Dijk y Kintsch 83, pp. 309-311), se puede utilizar como punto de partida para el estudio de algunas de las dificultades de aprendizaje que plantean los textos científicos. 


\section{LOS ESQUEMAS EN EL APRENDIZAJE DE LA CIENCIA: PROBLEMAS DE COMPRENSION}

Los problemas en torno a la utilización de esquemas en el aprendizaje de textos científicos pueden ser, al menos, de las dos clases que se consideran a continuación. Kieras (85) apunta la primera clase de problemas refiriéndose a la comprensión de prosa técnica:

"Sin embargo, la característica principal de la prosa técnica es que, en general, su contenido es básicamente nuevo para el que lee, por ejemplo un libro de texto, y por tanto no se pueden aplicar de manera apreciable esquemas de contenido. Esto sugiere que la comprensión de prosa técnica no es fundamentalmente una cuestión de concordancia entre el contenido y un patrón conocido previamente, sino que implica tratar con el contenido del pasaje al nivel de las proposiciones individuales que expresan el contenido (p.94).

Por tanto, es posible que el que aprende ciencias no disponga de esquemas adecuados para comprender la información que se le presenta. Puede intentar tratar el contenido "al nivel de las proposiciones individuales", pero también puede que intente activar esquemas inadecuados. La siguiente situación ilustra este problema. Se dio una serie de textos breves a un grupo de alumnos de ciencias del Curso de Orientación Universitaria, indicándoles que los leyesen hasta comprenderlos lo suficientemente bien como para estar en condiciones de explicárselos a sus compañeros. Se trataba de evitar que utilizasen criterios de comprensión relajados que les llevasen a una lectura superficial del texto. Se solicitaba a los alumnos, inmediatamente después de leer el texto, que explicasen su contenido al entrevistador y que pidiesen cualquier aclaración necesaria si encontraban partes del textos oscuras o que no entendiesen bien. Con la prueba se intentaba explorar el grado en que los alumnos comprendían la información que se le proporcionaba y el grado en que eran conscientes de que entendían o no entendían el texto. Uno de estos textos era el siguiente:

"El término gota fría corresponde a una situación atmosférica de embolsamiento de aire frío en altura. La masa de aire, aislada como una burbuja, tiende a descender hacia el suelo. La mezcla de aire frío con el entorno más cálido es lo que provoca abundantes lluvias, sobre todo en los bordes de la gota. Por esta razón, en la zona mediterránea la gota fría se suele traducir en lluvias torrenciales. Más infrecuente es que la gota fría aparezca sobre el Cantábrico, por lo general de aguas frías".

La comprensión de este tex to implica activar un esquema relacionado con condensación del vapor de agua. Una de las posibles variables del esquema condensacion se refiere a la temperatura. La información "la mezcla de aire frío con el entorno más cálido" se comprende en este texto porque corresponde a la variable temperatura, es decir, permite asignar en este caso concreto un valor a esta variable: la temperatura del aire cálido ha disminuido (3) (conduciendo a la condensa- ción del vapor de agua existente en él y, finalmente, a las lluvias). Si no se utiliza este esquema, es muy posible que en el esquema alternativo que se active sea difícil o imposible asignar esta información a casilla al. guna. Éste es el problema que parecía encontrar la alumna siguiente (SBr387P1):

Alumna SBr387P1: ¿Es embolsamiento de aire frío?

Entrevistador: Embolsamiento de aire frío... ¿qué es lo que no entiendes de eso?

A: Pues que sea aire y luego lo que produce son lluvias.

A: Entonces produce lluvias pero... las lluvias se supone que están ahí ya, o sea... las nubes... son propensas a tener liuvia... están ahí... viene el aire frío y...

$\mathrm{E}:$ ¿Tu pega cuál es?

A: Mi pega es esa... como el aire produce lluvias si tiene que ser agua.

$\mathrm{E}$ : Como el aire produce lluvia si tiene que ser agua...

A: Quiero decir que... que el aire frío lo que pasa es que embolsa una masa de agua... de nubes... o...

Esta alumna parece entender que la lluvia se produce a partir del agua existente en las nubes sin que en este proceso intervenga la temperatura. La alumna no podía entonces ubicar de manera fácil la información sobre el aire frío en ninguna de las casillas de su esquema. Se daba cuenta de ello, y por tanto reconocía que tenía problemas de comprensión.

\section{LOS ESOUEMAS EN EL APRENDIZAJE DE LA CIENCIA: PROBLEMAS DE META- COMPRENSION}

Existe una segunda clase de problemas relacionados con el uso de los esquemas. En el caso de los textos científicos el sujeto puede carecer de esquemas adecuados, como se acaba de mencionar, y ser consciente de que no puede organizar convenientemente las proposiciones del texto. Otro comportamiento consiste en activar, entre los posibles esquemas, uno inadecuado y no ser consciente de que lo es. El sujeto utiliza un esquema que no admite en sus casillas la información que se desprende del texto pero no es consciente del desajuste y cree que comprende bien. El siguiente protocolo ilustra este tipo de actuación:

Alumno SBr387E1: Eeeeh... es que... habla de situación atmosférica pero habla de temperatura, entonces por diferencia de temperatura se... eeeeh... crean... eeeeeh... unas altas y bajas presiones... entonces... eeeeh... esto es lo que provoca... eeeeh... Ios anticiclones y las borrascas, entonces... eeeeh... esto... la diferencia entre altas y bajas presiones es lo que provoca... pues, por así decirlo, esta gota fría. 
Puede resultar sorprendente que inmediatamente después de leer el párrafo anterior un alumno pueda dar una versión tan distorsionada del contenido del texto. La contestación del alumno se puede interpretar suponiendo que ha aplicado los esquemas que tenía dispo. nibles en relación con el conteniđo del texto. El alumno tampoco activa esquema alguno relacionado con la condensacion. Parece haber un esquema general relacionado con el tiempo, sugerido, quizá, por la mención de "aire", "situación atmosférica" o "lluvias". Lo que interesa resaltar es que, a diferencia del caso anterior, el alumno no se da cuenta de que el esquema no es adecuado para explicar la información que se le presenta. No hace preguntas sobre el significado del texto, pese a haber instrucciones de que lo hiciese si tenía algún problema de comprensión. Esta actuación se pone de manifiesto también cuando el alumno lee un segundo texto, en el que se había introducido delibera. damente un error:

Viajando a $60 \mathrm{Km} / \mathrm{h}$ se tardan 15 minutos en llegar a un lugar. A la vuelta se viaja a $40 \mathrm{Km} / \mathrm{h}$. El tiempo que se tarda en este caso es:

$$
X=\frac{40 \cdot 15}{60}=10 \text { minutos }
$$

A: ... Es una relación velocidad tiempo. Entonces... pues a más velocidad lógicamente... eeeh... se invierte menos tiempo... es una relación directa.

Tampoco aquí pide aclaración alguna ni iđentifica el error existente en el texto. El alumno utiliza su esquema de "proporción inversa" (aun cuando declara que se trata de una "relación directa"), válido para analizar las relaciones entre velocidad constante de un móvil y el tiempo que invierte en realizar un recorrido: a más velocidad menos tiempo. No nota que el dato de menos velocidad y menor tiempo invertido en el recorrido no puede ubicarse en este esquema, sino que correspondería a otro de "proporción directa". Ello pone de manifiesto los procesos "arriba-abajo" o "controlados conceptualmente" en la comprensión del texto. El alumno activa un esquema que le guía en la comprensión permitiéndole, incluso, prescindir de parte de la información que le proporciona el texto ("menor velocidad") porque puede ser suplida por defecto: si en la casilla correspondiente a una de las magnitudes, el tiempo, hay una disminución, en la casilla correspondiente a la otra, la velocidad, debe haber un aumento. El texto indica una disminución pero el sujeto no consideró necesario recurrir a un proceso "abajo-arriba" o "controlado por los datos" para comprobarlo.

Los problemas que se acaban de apuntar son de naturaleza metacognitiva. En la primera de estas dos contes. taciones no se trata solamente de que el alumno no entiende una información correcta, sino que no se da cuenta de que no entiende. No controla adecuadamente su comprensión. En el segundo caso "entiende correctamente" una información incorrecta, con errores que debiera detectar. La explicación đe estas actuaciones remite a un problema fundamental:

"Quizá las preguntas centrales en el desarrollo de un modelo de la percepción y la comprensión basado en esquemas son: Primero, icómo se descubre una configuración adecuada de esquemas? Segundo, ¿cómo se evalúa la bondad de ajuste? (Rumelhart 80, p. 41 Subrayado añadido).

¿Por qué hay alumnos sensibles a las discrepancias entre las exigencias del esquema y la información que proporciona el texto y otros que no lo son? Como indica Rumelhart, ¿de qué manera evalúan los individuos la bondad de ajuste del esquema a los datos? Conocer las respuestas a estas preguntas ayudaría a entender una fuente importante de dificultades en el aprendizaje de la ciencia.

\section{IMPLICACIONES PARA LA ENSEÑANZA DE LA CIENCIA}

La ausencia de esquemas que permiten comprender la información científica que se proporciona a los alumnos es un problema relativamente conocido por las personas dedicadas a la enseñanza de la ciencia. Como se apunta más arriba, ha existido una preocupación importante en los últimos años por conocer las preconcepciones con las cuales inician los alumnos el aprendizaje de la ciencia. Sin embargo no se han considerado todavía con el sufuciente detalle la segunda clase de problemas, metacognitivos. El grado de comprensión alcanzado depende, en último término, de una decisión del que aprende, independientemente del significado lógico de los materiales de enseñanza que proporcione el profesor. Algunos alumnos creen que comprenden adecuadamente lo que es una gota fría si no encuentran problemas de léxico. Otro cree que el tex to informa de la existencia de borrascas y anticiclones causadas por la diferencia de temperaturas. Es muy difícil que puedan astmilar la información que intenta proporcionar el texto (la explicación del mecanismo de producción de lluvias abundantes) si no son conscientes de que hay algo que les falta por entender. En contra de esta conclusión se podría argumentar que los problemas planteados podrían evitarse con una confección más cuidadosa del texto, adecuándolo al nivel de conocimiento de los alumnos. $\mathrm{Si}$, en este caso, se hiciese explícito el mecanismo de producción de lluvias (4), en lugar de mantenerlo implícito, habría una menor posibilidad de que el alumno creyese que entiende, cuando no es así. De nuevo se intentaría encontrar la solución en la mejora del significado de los materiales. Sin embargo, nunca se podría estar seguro de que los materiales conectan adecuadamente con ideas apropiadas que ya posea cada alumno. Y si hay un salto entre la información que se proporciona y las ideas, o esquemas, que posee el que aprende, se vuelve de nuevo a una parcela del aprendizaje cuyo control parece estar únicamente en las manos de uno mismo. 
Los problemas anteriores no deberían, sin embargo, conducir al abandonismo suponiendo que el control de la propia comprensión es un reducto inaccesible a la enseñanza. El fenómeno se viene considerando desde hace algún tiempo por los investigadores en el área de Ia lectura, por ejemplo, llevando al diseño de currículos experimentales para el desarrollo de destrezas meta. cognitivas que mejoren la comprensión lectora (Paris, Cross y Lipson 84). No parece imposible un trabajo con los mismos objetivos en el área de la ciencia; de hecho, se han realizado ya algunos (Baird, 86). Es muy posible que estemos solamente en el inicio de una línea de estudio con importancia creciente en el futuro.

\section{NOTAS}

(1) Aunque no ha intentado explicar con detalle la organizacion del conocimiento en la memoria del que aprende.

(2) Ejemplo tomado de Bransford y Stein (84). La investigación de Bransford y sus colaboradores es una fuente de ejemplos llamativos que ponen de manifiesto fenómenos importantes del aprendizaje.

\section{REFERENCIAS BIBLIOGRÁFICAS}

AGUIRRE DE CÁRCER, I., 1983. Dificultades en la comprensión de las explicaciones de los libros de texto de Física, Enseñanza de las Ciencias, 1(2), pp. 92-98.

AUSUBEL, D., 1968. Educational Psychology: A cognitive View. (New York: Holt, Rinehart and Winston). (Traduc. cast. Ed. Trillas, 1976).

AUSUBEL, D., NOVAK, J. y HANESIAN, H., 1978. Educational Psychology. A cognitive View. (New York: Holt, Rinehart and Winston). (Traduc. cast. Ed. Trillas, 1983).

BAIRD J. R., 1986. Improving learning through enhanced metacognition: a classroom study, European Journal of Science Education, 8(3), pp. 263-268.

BAKER, L. (en prensa). Metacognition, Reading, and Science Education, en Science Learning: Processes and Applications, (Newark, DE: International Reading Association).

BARTLETT, F.C., 1932. Remembering. A Study in Experimental and Social Psychology. (Cambridge: Cambridge University Press). (2a reimpresión, 1977).

BRANSFORD, J.D. y STEIN, B.S., 1984. The ideal problem solver. (New York: W.H. Freeman). (Traduc. cast. Ed. Labor, 1986).

BROWN, A.L., 1980. Metacognitive Development and Reading, en Spiro, R.J., Bruce, B.C. y Brewer, E.F. (eds.). Theoreticallssues in Reading Comprehension. (Hillsdale, N.J.: Lawrence Eribaum).

\section{Agradecimiento}

Este trabajo es parte de una investigación subvencionada por el CIDE durante el periodo 1986-88. Agradece. mos a D. Raimundo Pascual, Catedrático de Física y Química del I.B. "Santa Brígida" de Madrid, la ayuda prestada para llevar a cabo las entrevistas utilizadas en el estudio. En su realización participaron también $\mathrm{E}$. Arribas, E. Lowy y D. Reyman.

(3) Este sería un valor adecuado para la variable del esquema. No sería aceptable, por supuesto, como valor concreto de la variable temperatura en el contexto de un problema físico.

(4) Hasta donde pudiese hacerse explicito un fenómeno relativamente complejo como éste.

BROWN, A.L., 1987. Metacognition, Executive Control, Self-Regulation and Other More Mysterious Mechanisms, en Weinert, F.E., Kiuwe, R.H. (eds.). Metacognition Motivation, and Understanding, (Hillsdale, N.J.: Lawrence Erlbaum).

BULLEJOS DE LA HIGUERA, J., 1983. Análisis de actividades en textos de Física y Química de $2^{\circ}$ de BUP, Enseñanza de las Ciencias, 3, pp. 147-157.

van DIJK T.A. y KINTSCH, W., 1983. Strategies of Discourse Comprehension. (New York: Academic Press).

DORAN, R. y SHEARD, D., 1974. Analyzing Science Textbooks, School Science and Mathematics, 74, pp. 31-39.

FERNÁNDEZ URÍA, E., 1978. Análisis de libros de texto de Ciencias en el Bachillerato, Revista de Bachillerato, 6 , pp. 10-14.

FINLEY F.N., 1983. Student's recall from science text, Jour nal of Research in Science Teaching, 20(3), pp. 247-259.

FlavelL, J, H., 1976. Metacognitive Aspects of Problem Solving, en L.B. Resnick (ed.), The Nature of Intelligence. (Hitlsdale, N.J.: Lawrence Erlbaum Ass).

HARMS, N., 1981. Project Synthesis: Summary and implications for teachers, en N.C. Harms y R.E. Yager (eds.), What Research Says to the Science Teacher. (Washington: National Science Teachers Association). 
KIERAS, D.E., 1985. Thematic Processes in the Comprehension of Technical Prose, en B.K. Britton y I.B. Black (eds.) Understanding Expository Text. (Hillsdale, N.J.: Lawrence Erlbaum).

MAYER, R.E., 1985a. Structural Analysis of Science Prose: Can we Increase Problem Solving Performance? en B.K. Britton y J.B. Black (eds.), Understanding Expository Text. (Hillsdale, N.J.: Lawrence Erlbaum Ass).

MAYER R.E., 1985b. How to Analyze Science Prose, en B.K. Britton y J.B. Black (eds.), Understanding Expository Text. (Hillsdale, N.J.: Lawsence Erlbaum Ass).

MINSKY, M., 1975. A framework for representing knowledge, en P. Winston (ed.), The psychology of computer vision. (New York: McGraw-Hill).

PARIS, S.G., CROSS, D.R. y LIPSON, M.Y., 1984. Informed strategies for learning: a program to improve reading awareness and comprehension, Journal of Educational Psychology, 76, pp. 1239-1252.

PROSSER, M., 1979. Cognitive Analysis of Physics textbooks at the Tertiary or College Level, Science education, 63(5), pp. 677-683.

RUMELHART, D.E, 1980. Schemata: the building blocks of cognition, en R. Spiro, B. Bruce, y W. Brewer (eds.),
Theoretical issues in reading comprehension. (Hillsdale, N.J.: Lawrence Erlbaum).

RUMELHART, E. y ORTONY, A., 1977. The repreșentation of knowledge in memory, en R.C. Anderson, R.J. Spiro y W.E. Montagne (eds.), Schooling and the acquisition of knowledge. (Hillsdale, N.J.: Lawrence Erlbaum).

SCHANK, R.C. y ABELSON, R.P., 1977. Seripts, plans, goals and understanding. (Hillsdale, N.J.: Lawrence Erlbaum). (Traduc. cast. Ed. Paidós, 1988).

VELARDE, M.G. y OTERO, J., 1984. La enseñanza de la fisica en el bachillerato. Memoria de Investigación. CIDE.

WANDERSEE, J.H., 1988. Ways students read text, Journal of Research in Science Teaching, 25(1), pp. 69-84.

WEISS, 1., 1978. Report of the 1977 National Survey on Science, Mathematics, and Social Studies Education. Center for Educational Research and Evaluation, Research Triangle Institute. Research Triangle Park, EEUU.

WILLIAMS, R.L. y YORE, L.D., 1985. Content, format, gender, and grade level differences in elementary students' ability to read science materials as measured by the cloze procedure, Journal of Research in Science Teaching, 22(1), pp. 81-88. 